\title{
ESTIMULACIÓN DE GERMINACIÓN Y COLONIZACIÓN RADICULAR DE Diversispora trimulares POR FLAVONOIDES DE EXUDADOS RADICULARES DE Nicotiana glauca
}

\section{STIMULATION OF GERMINATION OF SPORES AND ROOT COLONIZATION OF Diversispora trimulares BY FLAVONOIDS IN Nicotiana glauca ROOT EXUDATES}

\author{
David Ramírez ${ }^{1}$, Blanca Naranjo ${ }^{2}$, Jéssica Duchicela ${ }^{3}$
}

${ }^{1}$ Egresado, email: dmramirez@espe.edu.ec; ${ }^{2}$ Dra. Química, M.Sc. Procesamiento de Alimentos, Docente investigador, email: bfnaranjo@espe.edu.ec; ${ }^{3}$ Ph.D. Ecología, Evolución y Comportamiento, Docente investigador, Departamento de Ciencias de la Vida y de la Agricultura. Universidad de las Fuerzas Armadas - Espe, P.O.BOX 1715231B, Av. General Rumiñahui s/n, Sangolquí, Ecuador, email: jiduchicela@espe.edu.ec

Rev. U.D.C.A Act. \& Div. Cient. 20(2): 341-351, Julio-Diciembre, 2017

\section{RESUMEN}

Los metabolitos secundarios son señales importantes en la interacción planta-microrganismos; sin embargo, los datos que corroboran el rol de los flavonoides como señales entre plantas y la simbiosis micorrízica arbuscular son limitados y aún recientes. Este estudio tiene por objetivo evaluar el efecto de flavonoides presentes en exudados radiculares de Allium porrum L., Nicotiana gluaca y Brassica oleracea var. Itálica, en la germinación de esporas y establecimiento de colonización radicular de Diversispora trimulares. Se detectaron miricetina y canferol en exudados de $A$. porrum, con un contenido total de flavonoides de $23,80 \mu \mathrm{g} \mathrm{g}^{-1}$, de raíz seca; quercetina, en $N$. glauca, con $23,35 \mu \mathrm{g} \mathrm{g}^{-1}$ y crisina, en $B$. oleracea, con $14,71 \mu \mathrm{g} \mathrm{g}^{-1}$. Quercetina estimuló la germinación y la vitalidad de esporas $(24 \%, 40 \%)$, a diferencia de crisina, que presentó un efecto inhibitorio (4\%, 20\%). A. porrum y $N$. glauca incrementaron la germinación de esporas $(54 \%, 56 \%)$ y porcentaje de colonización de micorriza arbuscular $(72 \%, 75 \%)$. Los resultados de la investigación mostraron una evidencia sólida del efecto de los flavonoides como moléculas estimulantes en los procesos de germinación de esporas y colonización de micorriza arbuscular.

Palabras clave: Micorriza arbuscular, exudados radiculares, flavonoides, germinación, quercetina.

\section{SUMMARY}

Secondary metabolites are important signals that could determine the outcome of the plant soil microbial interactions, however, the data available that support the leading role of flavonoids in the communication of arbuscular mycorrhizal symbiosis are recent and limited. The present study examines the effect of flavonoids detected in root exudates from Allium porrum, Nicotiana glauca y Brassica oleracea var. italica on spore germination and mycorrhizal root colonization of Diversispora trimulares. Myricetin and kaempferol were detected in A. porrum root exudates with a total flavonoid content of $23.80 \mu \mathrm{g} \mathrm{g}^{-1}$ dried root, quercetin in $N$. glauca with $23,35 \mu \mathrm{g} \mathrm{g}^{-1}$ and chrysin in B. Oleracea with $14.71 \mu \mathrm{g} \mathrm{g}^{-1}$. Quercetin increased the spore germination and vitality $(24 \%, 40 \%)$. In contrast, chrysin showed an inhibitory effect $(4 \%, 20 \%)$. A. porrum and $N$. glauca increased spore germination $(54 \%, 56 \%)$ and root mycorrhizal colonization (72\%, $75 \%)$. This results evidence the effect of flavonoids on the stimulization of spore germination and stablishment of root colonization of arbuscular mycorrhizal fungi.

Key words: Arbuscular mycorrhiza, root exudates, flavonoids, germination, viability

\section{INTRODUCCIÓN}

El sistema radicular libera un amplio rango de componentes, los cuales, lideran comunicaciones complejas en procesos de la rizósfera. Entre estos compuestos, se incluyen polisacáridos, aminoácidos, ácidos aromáticos, enzimas, reguladores del crecimiento y otros metabolitos secundarios. Adicionalmente, se conoce que los hongos del suelo responden a los exudados radiculares; sin embargo, el conocimiento del efecto de los metabolitos secundarios en la rizósfera, aún es limitado. 
El presente estudio, se enfoca en un tipo de exudado radicular, que ha sido identificado como clave en las señales moleculares de las interacciones entre plantas y hongos del suelo, los flavonoides (Cesco et al. 2010), metabolitos secundarios que se derivan de vías biosintéticas mixtas (Ferrer et al. 2008). En plantas, se han identificado diferentes flavonoides, que incluyen chalconas, flavonas, flavonoles, antocianinas, proantocianidinas y auronas (Ferrer et al. 2008). Se presentan en varias familias vegetales, entre las que se encuentran especies del género Solanum (Medina-Medrano et al. 2015); sin embargo, aún no se han reportado flavonoides presentes en exudados radiculares de Nicotiana glauca, miembro de la familia Solanaceae, especie vegetal naturalizada, presente en zonas áridas de los valles interandinos del Ecuador. Esta especie es utilizada como portainjerto para producción de frutales, por su resistencia a Fusarium sp. y nematodos, así como su potencial asociación con micorriza arbuscular (Viera et al. 2017).

El establecimiento de la simbiosis micorrízica, entre los hongos del Phylum Glomeromycota y $80 \%$ de las plantas vasculares (Smith \& Read, 2010), requiere una compleja comunicación entre las raíces de acogida y los hongos glomeromycetes (Vierheilig, 2004). Las primeras señales que perciben los glomeromycetes son la exudación de compuestos, a través de la raíz, entre los que se tiene a los flavonoides (Mierziak et al. 2014). Los reportes del efecto de los flavonoides en el desarrollo de micorriza arbuscular son contrastantes. Estudios realizados por Buee et al. (2000) re- portan que los exudados radiculares de Daucus carota indujeron a la germinación de esporas de Gigaspora gigantea; no obstante, Vierheilig (2004) analizó exudados de algunas especies de Brassicaceae que inhibieron la germinación y el desarrollo de hifas de esporas de Glomus mosseae. Estos estudios sugieren que los exudados radiculares pueden alterar la susceptibilidad de plantas a ser micorrizadas ya que tienen la capacidad de promover o inhibir la germinación de esporas, mientras que Ponce et al. (2004) identificaron flavonoides, como: quercetina, miricetina y canferol, en los exudados radiculares que interactúan en la simbiosis planta-micorriza. Scervino et al. (2005) y Lum \& Hirsch (2003) estudiaron estos metabolitos y reportaron que la influencia en la germinación y en el crecimiento de hifas de esporas de micorriza arbuscular está provocada por la presencia de un grupo hidroxilo en el $\mathrm{C}_{3}$ del núcleo del flavonoide. Además, Scervino et al. (2006) encontraron que quercetina (flavonoide con hidroxilos en $\mathrm{C}_{4}$ y $\mathrm{C}_{5}$ ) originó mayor germinación en comparación con miricetina (flavonoide trihidroxilado en $\mathrm{C}_{3}, \mathrm{C}_{4}, \mathrm{y} \mathrm{C}_{5}$ ) y canferol (flavonoide monohidroxilado en $\mathrm{C}_{4}$ ), mientras que crisina (flavonoide sin hidroxilos en $\mathrm{C}_{3}, \mathrm{C}_{4} \mathrm{y}^{\prime} \mathrm{C}_{5}$ ) inhibió la germinación de esporas (Tabla 1). Estos estudios sostienen que el efecto estimulante o inhibitorio de los flavonoides puede variar en dependencia de la especie vegetal que lo produce (Scervino et al. 2006) y de la concentración de flavonoides (Miean et al. 2001). El objetivo de este estudio fue determinar los flavonoides presentes en los exudados radiculares de $N$. glauca y evaluar su efecto en la germinación de esporas de $D$. trimulares.

Tabla 1. Estructuras de flavonoides encontrados en los exudados radiculares.

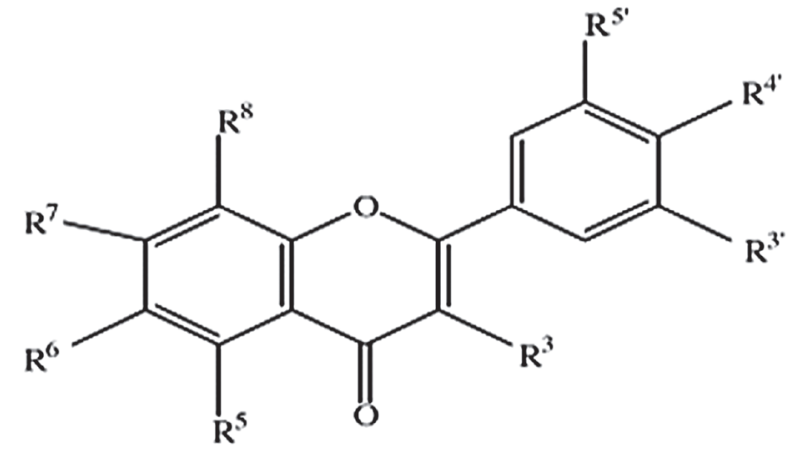

\begin{tabular}{|llllllllll}
\hline Crisina & $\mathrm{H}$ & $\mathrm{OH}$ & $\mathrm{H}$ & $\mathrm{OH}$ & $\mathrm{H}$ & $\mathrm{H}$ & $\mathrm{H}$ & $\mathrm{H}$ \\
\hline
\end{tabular}

\begin{tabular}{|l|l|l|l|l|l|l|l|l|}
\hline Compuesto & $\mathrm{R}^{3}$ & $\mathrm{R}^{5}$ & $\mathrm{R}^{6}$ & $\mathrm{R}^{7}$ & $\mathrm{R}^{8}$ & $\mathrm{R}^{3}$ & $\mathrm{R}^{4}$ & $\mathrm{R}^{5}$ \\
\hline Quercetina & $\mathrm{OH}$ & $\mathrm{OH}$ & $\mathrm{H}$ & $\mathrm{OH}$ & $\mathrm{H}$ & $\mathrm{H}$ & $\mathrm{OH}$ & $\mathrm{OH}$ \\
\hline Canferol & $\mathrm{OH}$ & $\mathrm{OH}$ & $\mathrm{H}$ & $\mathrm{OH}$ & $\mathrm{H}$ & $\mathrm{H}$ & $\mathrm{OH}$ & $\mathrm{H}$ \\
\hline Miricetina & $\mathrm{OH}$ & $\mathrm{OH}$ & $\mathrm{H}$ & $\mathrm{OH}$ & $\mathrm{H}$ & $\mathrm{OH}$ & $\mathrm{OH}$ & $\mathrm{OH}$ \\
\hline Crisina & $\mathrm{H}$ & $\mathrm{OH}$ & $\mathrm{H}$ & $\mathrm{OH}$ & $\mathrm{H}$ & $\mathrm{H}$ & $\mathrm{H}$ & $\mathrm{H}$ \\
\hline
\end{tabular}




\section{MATERIALES Y MÉTODOS}

Obtención de exudados radiculares. Semillas comerciales de Nicotiana glauca (tabaquillo), Allium porrum L. (cebolla) y Brassica oleracea var. italica (brócoli) fueron colocadas por separado en contenedores plásticos con sustrato estéril (tierra negra y cascajo relación 2:1). A los tres meses de edad, se extrajeron las plantas con sus raíces y se lavaron con agua corriente. Se introdujeron en Erlenmeyers con agua destilada y después de 48 horas, los exudados se recolectaron, se filtraron y se conservaron a $-14^{\circ} \mathrm{C}$, hasta su análisis (Prikryl \& Vancura, 1980).

Cuantificación de flavonoides totales. A través del método espectrofotométrico, descrito por Zhishen et al. (1999), se determinó el contenido total de flavonoides, usando rutina como estándar $\left(0,1 \mathrm{mg} \mathrm{mL}^{-1}\right)$, a una longitud de onda de $510 \mathrm{~nm}$ y se expresaron los resultados en $\mu \mathrm{g}$ de rutina $\mathrm{g}^{-1} \mathrm{de}$ raíz seca.

Preparación de la curva de calibración. Aplicando el método reportado por Zhishen et al. (1999), se elaboró la curva de calibración de flavonoides, usando una solución estándar de rutina. Se emplearon diferentes volúmenes de esta solución $(100,300,500,700,850$ y $1000 \mu \mathrm{L})$ y se completó con metanol hasta un volumen de $1250 \mu \mathrm{L}$; se adicionó $75 \mu \mathrm{L}$ de nitrito de sodio $\left(\mathrm{NaNO}_{2}\right)$ al $5 \%$, reposo de $6 \mathrm{~min}$ y $150 \mu \mathrm{L}$ de cloruro de aluminio $\left(\mathrm{AlCl}_{3}\right)$ al $10 \%$, con $500 \mu \mathrm{L}$ de hidróxido de sodio $(\mathrm{NaOH}) 1 \mathrm{~N}$; finalmente, se completó con agua destilada hasta un volumen de 2,5mL. La medición, se realizó en espectrofotómetro (UV-VIS Genesys 10S), a una longitud de onda de 510nm.

Análisis de muestras. Las muestras, se prepararon siguiendo el método de Zhishen et al. (1999), sobre $500 \mu \mathrm{L}$ de cada exudado, en tubos de ensayo. Se evaluó la absorbancia a $510 \mathrm{~nm}$, en un espectrofotómetro (UV-VIS Genesys 10S). Se cuantificó los flavonoides exudados por gramo de raíz y se expresó en microgramos equivalentes de rutina por gramo de muestra seca.

Análisis de los exudados radiculares por cromatografía líquida de alta resolución (HPLC). La identificación de los flavonoides presentes en los exudados radiculares de $A$. porrum, N. glauca y $B$. oleracea, se analizaron mediante HPLC, en un equipo Varian ProStar 320 y como estándares se usaron: naringina, hesperidina, diosmina, catequina, quercetina, miricetina, daidzeina, canferol, apigenina, morina, hidroxiflavona, baicaleina, luteolina, naringenina, genisteina, hiperósido, crisina, epigalocatequina, silibina y rutina. Las muestras, se prepararon por filtración con membranas milipore $(0,45 \mu \mathrm{m})$, previo al análisis cromatográfico. La fase móvil estuvo constituida de una mezcla de solventes: A (agua-ácido fórmico 95:5) y B (metanol) y una fase estacionaria: sílica de fase reversa $C_{18}$, en columna de 150 x 4,6mm (Varian), diámetro de partícula de $5 \mu \mathrm{m}$. El gradiente de solventes A y B fue 65:35 - 25min, 50:50 - 10min, 20:80 - 5min, 10:90 $-5 \mathrm{~min}$ y $65: 35-15 \mathrm{~min}$. Se utilizó un flujo de $1,0 \mathrm{~mL} \mathrm{~min}^{-1}$, examinado a $280 \mathrm{~nm}$, con un volumen de inyección de $20 \mu \mathrm{L}$. Se analizó el tiempo de retención de las bandas de absorción de cada exudado radicular y se comparó con los estándares.

Dilución de exudados radiculares. Para aplicar los tratamientos, se tomó el exudado radicular puro, como concentración de $100 \%$ y, a partir de éste, se realizó diluciones al 50,20 y $0 \%$.

Diseño experimental. Las variables experimentales la constituyeron los exudados radiculares provenientes de $A$. porrum, N. glauca, B. oleracea, aplicados por separado, en tres concentraciones de exudado radicular $(0,20,50,100 \%)$, resultando 12 tratamientos, los cuales, se ubicaron siguiendo un diseño completamente al azar factorial $3 \times 4$, con tres réplicas y originando 36 unidades experimentales, donde los tratamientos con A. porrum constituyeron el control positivo (Mukherjee \& Ane, 2011), B. oleracea, el control negativo (Javaid, 2007) y agua estéril, el control absoluto.

Experimento 1. Evaluación de las concentraciones de flavonoides de exudados radiculares en la germinación de esporas de $D$. trimulares, en condiciones de laboratorio. Se colocaron 40 esporas de $D$. trimulares en membranas milipore dentro de una caja Petri, que contenía $20 \mathrm{~g}$ de arena estéril; $2 \mathrm{~mL}$ de cada exudado se aplicaron en las cajas. Los tratamientos, se colocaron conforme al diseño experimental descrito y, finalmente, las cajas se sellaron e incubaron en oscuridad a $27^{\circ} \mathrm{C}$, por 30 días.

Experimento 2. Evaluación de las concentraciones de flavonoides de exudados radiculares en la colonización radicular de $D$. trimulares en plántulas de Avena sativa, en condiciones de invernadero. Se utilizó $100 \mathrm{~g}$ de un inóculo de $D$. trimulares de 4 esporas/g seco de sustrato, colocado en $400 \mathrm{~g}$ de suelo estéril, en contenedores plásticos. En cada contendor, se ubicó una plántula de 30 días de edad de Avena sativa, los tratamientos se aplicaron siguiendo un diseño de bloques completamente al azar, colocando $40 \mathrm{~mL}$ de exudado en las unidades experimentales. El experimento, se mantuvo en condiciones de invernadero, a una temperatura de $21^{\circ} \mathrm{C}$ y con humedad relativa del $62 \%$, por 60 días.

Para determinar el porcentaje de germinación de esporas de micorrizas, se observaron las esporas de micorriza arbuscular a los 3, 5, 10, 15 y 30 días, posteriores a la aplicación de los exudados, con la ayuda de un estéreo-microscopio Olympus SZ61. Se consideró una espora germinada cuando presentaba crecimiento del tubo germinativo. La evaluación de este índice en el experimento in vitro, se realizó colocando 
las cajas petri directamente en el estéreo-microscopio. Para el experimento en invernadero, se tomó $50 \mathrm{~mL}$ de sustrato del contenedor y se aisló las esporas mediante el método de tamizado húmedo, de Gerdemann \& Nicolson (1963). La cuantificación del número de esporas, se realizó utilizando el estéreo-microscopio.

La vitalidad de esporas, se determinó al finalizar el experimento, utilizando el método de tinción vital (Bansal et al. 1991), como se describe. Se preparó una solución madre de $0,5 \mathrm{mM}$ de diacetato de fluoresceina (FDA), adicionando $1,04 \mathrm{mg}$ de FDA en $5 \mathrm{~mL}$ de dimetilsulfóxido (DMSO), sonicando por una hora hasta disolución completa y almacenándola en congelación, a $-14^{\circ} \mathrm{C}$. La solución de ensayo fue preparada con $0,25 \mathrm{~mL}$ de $\mathrm{FDA}$, aforada a $5 \mathrm{~mL}$ de una disolución $60 \mathrm{mM}$ de buffer fosfato salino (PBS), a un pH de 7,4, obteniendo una concentración de $25 \mu \mathrm{M}$; se sonicó durante una hora y se filtró en membranas milipore de $0,2 \mu \mathrm{m}$. Las esporas de cada tratamiento, se colocaron en un vidrio reloj y se añadió $5 \mu \mathrm{L}$ de la solución de ensayo, se dejó reposar por $15 \mathrm{~min}$ en oscuridad; luego, las esporas fueron colocadas en un portaobjetos con una gota de PBS y observadas en un microscopio de fluorescencia (Olympus IX53), con un filtro de excitación de 450-490nm y un filtro de emisión de $520 \mathrm{~nm}$. Se realizaron tres réplicas por cada tratamiento, las esporas que fluorescieron en color verde fueron tomadas como esporas vivas y las que no tuvieron fluorescencia, se consideraron muertas.

El porcentaje de colonización del experimento en invernadero fue determinado utilizando una muestra fresca de raíz de cada uno de las unidades experimentales, las cuales, fueron teñidas, empleando el método de Phillips \& Hayman (1970). En un portaobjetos y con agujas de disección, se colocaron segmentos de raíz de, aproximadamente, $1 \mathrm{~cm}$, en forma paralela, hasta cubrir toda la placa y se observó en microscopio óptico, siguiendo el método descrito por McGonigle et al. (1990); se realizaron tres réplicas por cada tratamiento. El porcentaje de colonización, se calculó a partir del número de campos positivos (presencia de hifas, esporas, arbúsculos y vesículas), sobre el número de campos visuales totales, multiplicados por 100.

Análisis estadístico. Se realizó una prueba de bondad de ajuste para comprobar que los datos sigan una distribución normal. Se llevó a cabo un análisis de varianza ANOVA (Analysis of Variance, según terminología inglesa), con el software estadístico INFOSTAT, para observar las diferentes respuestas en función de la presencia o ausencia del exudado radicular. Se utilizó la prueba de LSD (Least Significant Difference, según terminología inglesa) de Fisher ( $p>0,05)$, cuando se registraron diferencias estadísticas para los tratamientos.

\section{RESULTADOS Y DISCUSIÓN}

Cuantificación de flavonoides totales. El contenido total de flavonoides expresados en $\mu \mathrm{g}$ de rutina por $\mathrm{g}$ de muestra seca, detectados en exudados de $N$. glauca fue de 23,35 $\mathrm{SD} \pm 0,57 \mu \mathrm{g} \mathrm{g}^{-1} ;$ A. porrum fue de $23,80 \mathrm{SD} \pm 1,16 \mu \mathrm{g} \mathrm{g}^{-1} \mathrm{y}$ en $B$. oleracea $14,71 \mathrm{SD} \pm 1,53 \mu \mathrm{g} \mathrm{g}^{-1}$. El exudado radicular de $N$. glauca presentó concentraciones de flavonoides totales similar a $A$. porrum y al comparar con $B$. oleracea, aumento 1,5 veces; este resultado está dentro de lo esperado, ya que $B$. oleracea es el control negativo. Miean et al. (2001) reportaron algunas especies vegetales, como $B$. oleracea, con $197,0 \mu \mathrm{g} \mathrm{g}^{-1}$; A. sativum, con $957,0 \mu \mathrm{g} \mathrm{g}^{-1}$ y S. melongena, con $219,5 \mu \mathrm{g} \mathrm{g}^{-1}$ de muestra seca. Estos resultados, se obtuvieron de extractos de plantas; sin embargo, se observa que el contenido de flavonoides es mayor que el detectado en los exudados radiculares, confirmando que la cantidad de estos metabolitos difiere según la parte de planta de donde se obtienen y la especie analizada.

Dilución de exudados. Para aplicar los tratamientos las diluciones de los exudados a concentraciones $(100,50,20$ y $0 \%$ ) fueron equivalentes a concentraciones de flavonoides de N. glauca $\left(23,35 ; 11,68 ; 4,67\right.$ y $\left.0 \mu \mathrm{g} \mathrm{g}^{-1}\right)$, A. porrum $(23,80$; 11,$90 ; 4,76$ y $\left.0 \mu \mathrm{g} \mathrm{g}^{-1}\right)$ y $B$. oleracea var. italica $(14,71 ; 7,36$; 2,95 y $\left.0 \mu \mathrm{g} \mathrm{g}^{-1}\right)$.

Análisis de los exudados radiculares por HPLC. Comparando los tiempos de retención (tR) de los cromatogramas a las condiciones descritas y comparadas con los estándares de flavonoides y los exudados radiculares, $N$. glauca mostró presencia de quercetina ( $\left.t_{R}=6,58 \mathrm{~min}\right) ; A$. porrum presencia de miricetina $\left(t_{R}=4,54\right)$ y canferol $\left(t_{R}=4,93 \mathrm{~min}\right)$. Danesh et al. (2016) mencionan la influencia de quercetina, como un flavonoide estimulante de la germinación; mientras que Cesco et al. (2012) y Ponce et al. (2004) reportaron, con la misma actividad, a miricetina y canferol. En $B$. oleracea var. italica, se reportó una banda de absorción, a un $t_{R}$ de 4,99min, correspondiente a crisina; Abdel-Lateif et al. (2012), la reportan como flavona que inhibe la germinación de esporas de Glomus sp.

Experimento 1. Evaluación de las concentraciones de flavonoides de exudados radiculares en la germinación de esporas de $D$. trimulares en condiciones de laboratorio.

Se observó que el porcentaje de germinación de esporas expuestas a exudados radiculares de $N$. glauca fue de $24 \%$ y de A. porrum, de $31 \%$. N. glauca $\left(23,35 \mu \mathrm{g} \mathrm{g}^{-1}\right)$ incrementó el porcentaje de germinación 6 veces, en comparación a $B$. oleracea $\left(14,71 \mu \mathrm{g} \mathrm{g}^{-1}\right)$ y 2,4 veces, en comparación con el control $\left(\right.$ ug g $\left.\mathrm{g}^{-1}\right)$. A. porrum, a la concentración de $23,80 \mu \mathrm{g}$ $\mathrm{g}^{-1}$, incrementó 2,8 veces el porcentaje de germinación de esporas frente al control. Se observa que a una concentración de $14,71 \mu \mathrm{g} \mathrm{g}^{-1}$ de $B$. oleracea, el porcentaje de ger- 

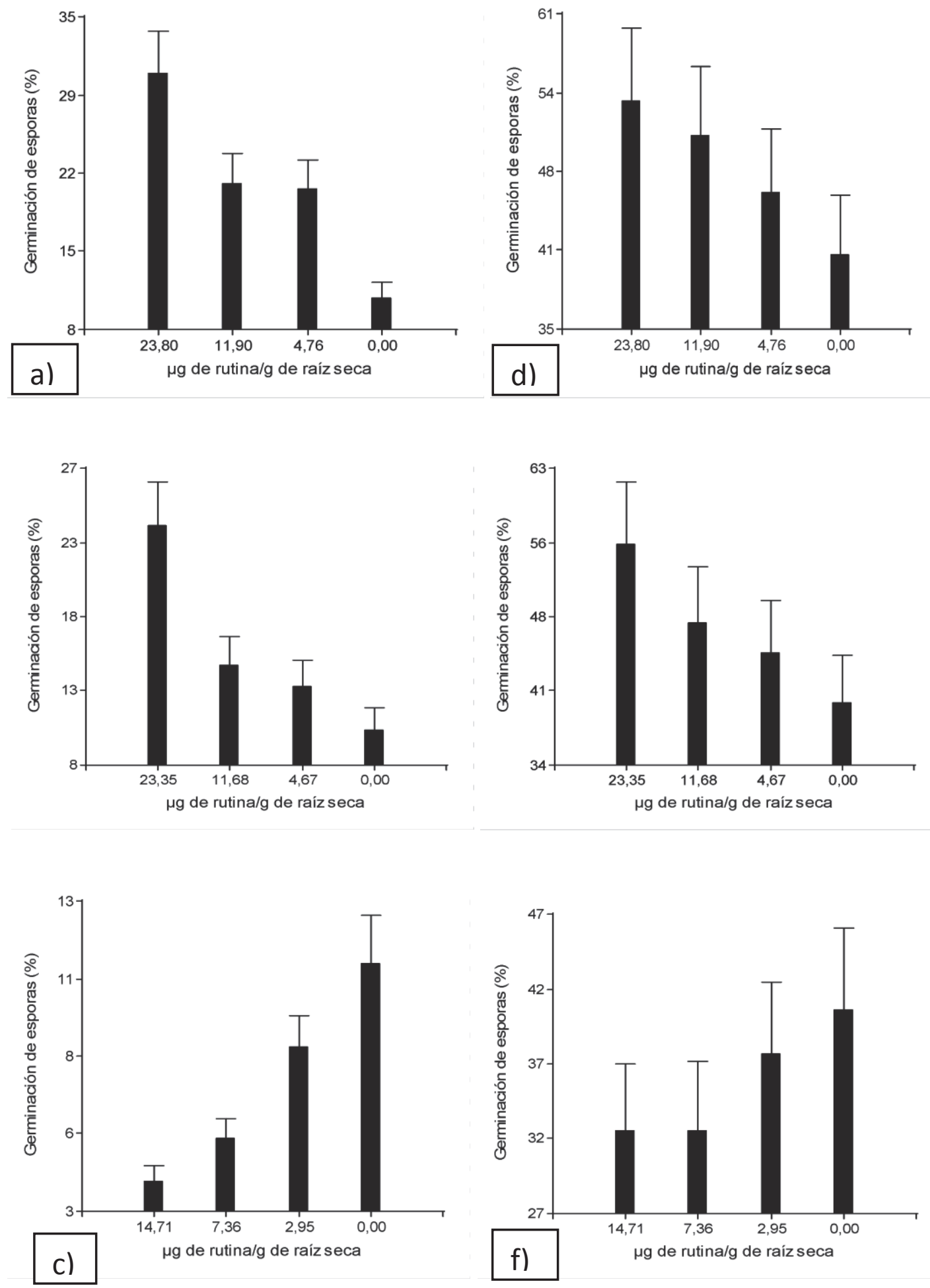

Figura 1. Porcentaje de germinación de esporas de $D$. trimulares, en presencia de exudados radiculares de: a) A. porrum; b) N. glauca; c) B. olerace, a en condiciones de laboratorio y d) A. porrum; e) $N$. glauca; f) B. oleracea, en condiciones de invernadero, a los 30 días de la aplicación de los tratamientos. 
minación disminuyó 2,7 veces, al comparar con el control (Figura 1a, 1b, 1c). Las diferencias observadas, se pueden atribuir a los flavonoides identificados en los exudados de N. glauca (quercetina) y A. porrum (miricetina y canferol), que incrementaron la germinación de esporas. Según Becard et al. (1992) y Lum \& Hirsch (2003), los hidroxilos en $\mathrm{C}_{3}, \mathrm{C}_{4}$ y $\mathrm{C}_{5}$ ' de la estructura de los flavonoides (quercetina, miricetina y canferol) producen un efecto estimulante en la germinación de esporas. En contraste con el efecto inhibidor producido por el exudado radicular de $B$. oleracea, que es atribuido al bajo contenido de flavonoides y la identificación de crisina, que según Scervino et al. (2006) es un flavonoide que no posee grupos hidroxilos en $\mathrm{C}_{3}, \mathrm{C}_{4}{ }^{\prime}$ y $\mathrm{C}_{5}$, por tanto, inhibe la germinación de esporas.

Según Requena et al. (2007), las señales químicas de los exudados, tales como los flavonoides que poseen estos hidorxilos son, posiblemente, reconocidos por proteinas receptoras de las micorrizas, que cambian e interactuan con componentes aguas abajo, como el factor Gin1, que se modifica mediante señales de la planta; a través de su actividad ATPasa, Gin1 puede transmitir señales al núcleo, lo que produce la transcripción de genes, que son fundamentales en la germinación y en la simbiosis.

La figura 2a revela el porcentaje de germinación de $D$. trimulares, comparado con el tiempo, desde la aplicación de los tratamientos. Se observó un incremento en la germinación de esporas durante los primeros 15 días; posterior a este período, la germinación de esporas no tuvo cambio significativo, según la prueba de LSD Fisher ( $p>0,05)$.

Las esporas a las que se aplicó los exudados radiculares de N. glauca y A. porrum respondieron positivamente a la tinción vital realizada (se tiñeron de color verde) y, en algunos casos, se pudo visualizar núcleos (Figura 3 ). El exudado de N. glauca $\left(23,35 \mu \mathrm{g} \mathrm{g}^{-1}\right)$ incrementó la vitalidad de esporas 2,2 veces, en comparación al control $\left(0,00 \mu \mathrm{g} \mathrm{g}^{-1}\right)$ y 4,4 veces, en comparación a $B$. oleracea $\left(14.71 \mu \mathrm{g} \mathrm{g}^{-1}\right)$. Al comparar con A. porrum no hubo diferencia significativa (Figura 4).

\section{Experimento 2. Evaluación de las concentraciones de flavonoides de exudados radiculares en la colonización radicular de $D$. trimulares, en plántulas de Avena sativa, en condiciones de invernadero.}

Los exudados de $N$. glauca reportan $56 \%$ de germinación a la concentración de $23,35 \mu \mathrm{g} \mathrm{g}^{-1}$, mayor que la reportada para A. porrum, que fue $54 \%$. N. glauca $\left(23,35 \mu \mathrm{g} \mathrm{g}^{-1}\right)$ incrementó el porcentaje de germinación de esporas 1,2 veces, al comparar con el control $\left(0.00 \mu \mathrm{g} \mathrm{g}^{-1}\right)$ y 1,8 veces, en comparación a $B$. oleracea $\left(14,71 \mu \mathrm{g} \mathrm{g}^{-1}\right.$ ) (Figura 1d, 1e, 1f). El porcentaje de germinación de $N$. glauca, en condiciones de invernadero, fue mayor que $A$. porrum; la diferencia entre estos resultados, se atribuye al efecto sinérgico que tiene la quercetina con el $\mathrm{CO}_{2}$, tal como lo reportan Chabot et al. (1992) y Becard et al. (1992), quienes probaron el efecto de flavonoides (quercetina, miricetina y canferol) en presencia de $\mathrm{CO}_{2}$ al $2 \%$, obteniendo el mayor porcentaje de germinación con quercetina, al ser comparado con miricetina y con canferol.

A todas las concentraciones estudiadas en el experimento en invernadero, se observó un incremento de tres veces el porcentaje de germinación, en comparación con los resultados observados en el experimento en condiciones de laboratorio. Según García et al. (2008), la presencia de $\mathrm{CO}_{2}$ estimula el crecimiento y la ramificación de hifas de esporas de micorrizas arbusculares. Adicionalmente, la presencia de minerales (N, K Y P) en el sustrato ayudan a estimular la capacidad biosintética de las esporas (Bücking et al. 2008). En la naturaleza, otro factor que influencia en la germinación de esporas es la interacción de micorrizas con las bacterias que se encuentran en el suelo, las cuales, estimulan la germinación, a través de la producción de factores de crecimiento y de destoxificación de sustancias antagónicas, que poseen las esporas (Frey-Klett et al. 2007). Este factor fue eliminado en el experimento, ya que se utilizó sustrato estéril.

En la figura $2 \mathrm{~b}$, se observa el porcentaje de germinación de $D$. trimulares, comparada con el tiempo después de la aplicación de los tratamientos, en condiciones de invernadero. Se observó un comportamiento similar al obtenido en condiciones de laboratorio; sin embargo, los porcentajes de germinación obtenidos fueron superiores.

El exudado de $N$. glauca $\left(23,35 \mu \mathrm{g} \mathrm{g}^{-1}\right)$ incrementó el porcentaje de colonización micorrízica de raíz 1,2 veces, en comparación al control y 1,9 veces, frente a $B$. oleracea $(14,71 \mu \mathrm{g}$ $\left.\mathrm{g}^{-1}\right)$. Comparando con A. porrum $\left(23,80 \mu \mathrm{g} \mathrm{g}^{-1}\right)$, no hubo una diferencia significativa (Figura 5). En los exudados radiculares se han identificado flavonoides (quercetina, miricetina, canferol), que son moléculas de señalización, para que la espora reconozca a la raíz y la pueda colonizar; sin embargo, Scervino et al. (2005) reportaron que, flavonas como crisina, impiden la colonización de raíces por Glomus mosae, Gigaspora rosea y Gi. margarita.

Del presente estudio, se concliuye que la eficiencia de colonización de hongos micorrízicos arbuscular a las plantas depende de la aptitud de las esporas de desarrollarse rápidamente, germinar en el suelo y producir colonización en su planta hospedera. Los resultados de la investigación mostraron una evidencia sólida del efecto de los flavonoides, como estimulantes de la germinación de esporas y proceso de colonización de micorriza arbuscular. En particular, quercetina, detectada en exudados radiculares de $N$. glauca, miricetina y canferol en exudados de A. porrum, estimularon la germina- 

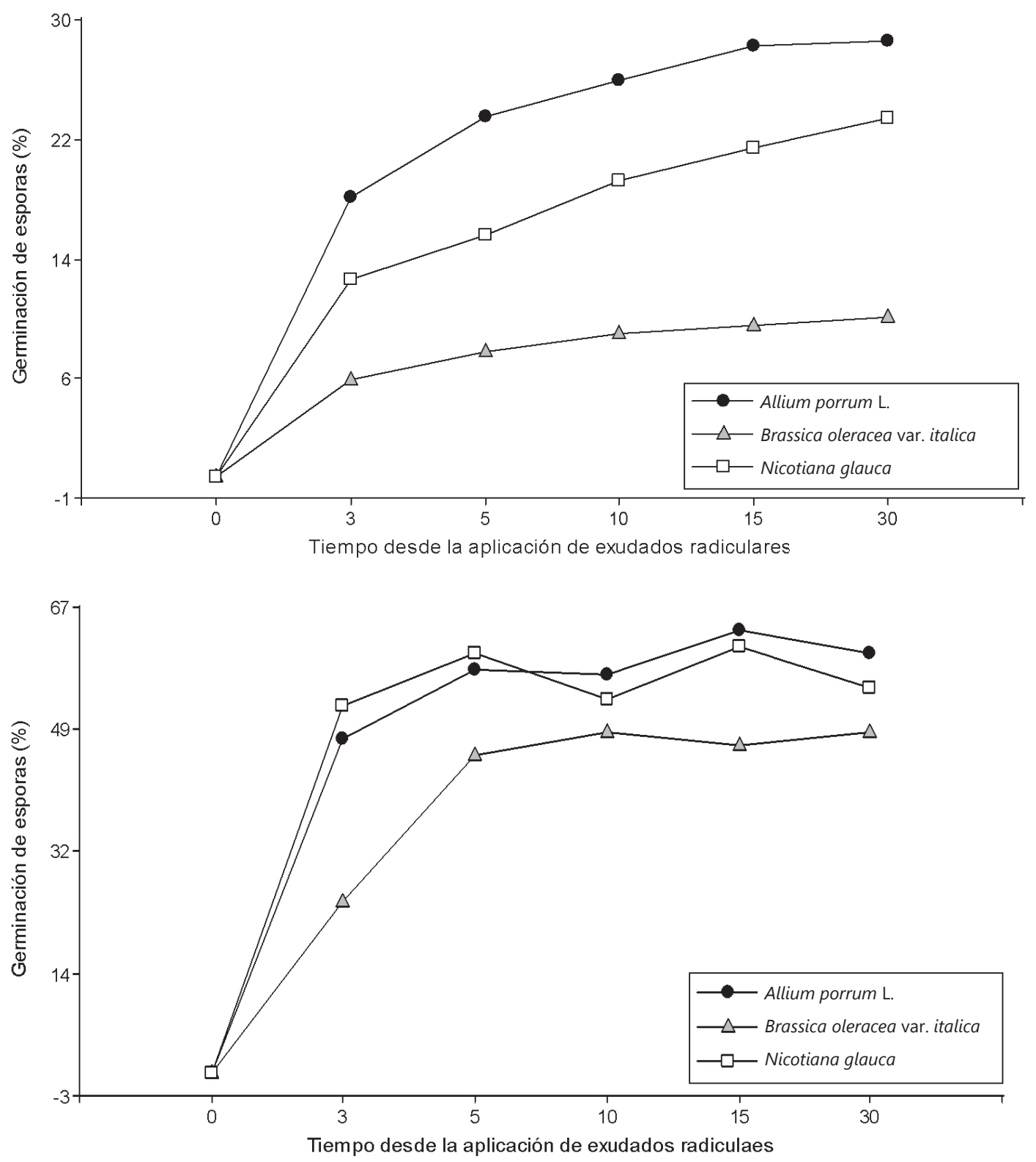

Figura 2. Porcentaje de germinación de Diversispora trimulares comparada con el tiempo, desde la aplicación de exudados radiculares. a) Condiciones de laboratorio y b) Condiciones de invernadero. 

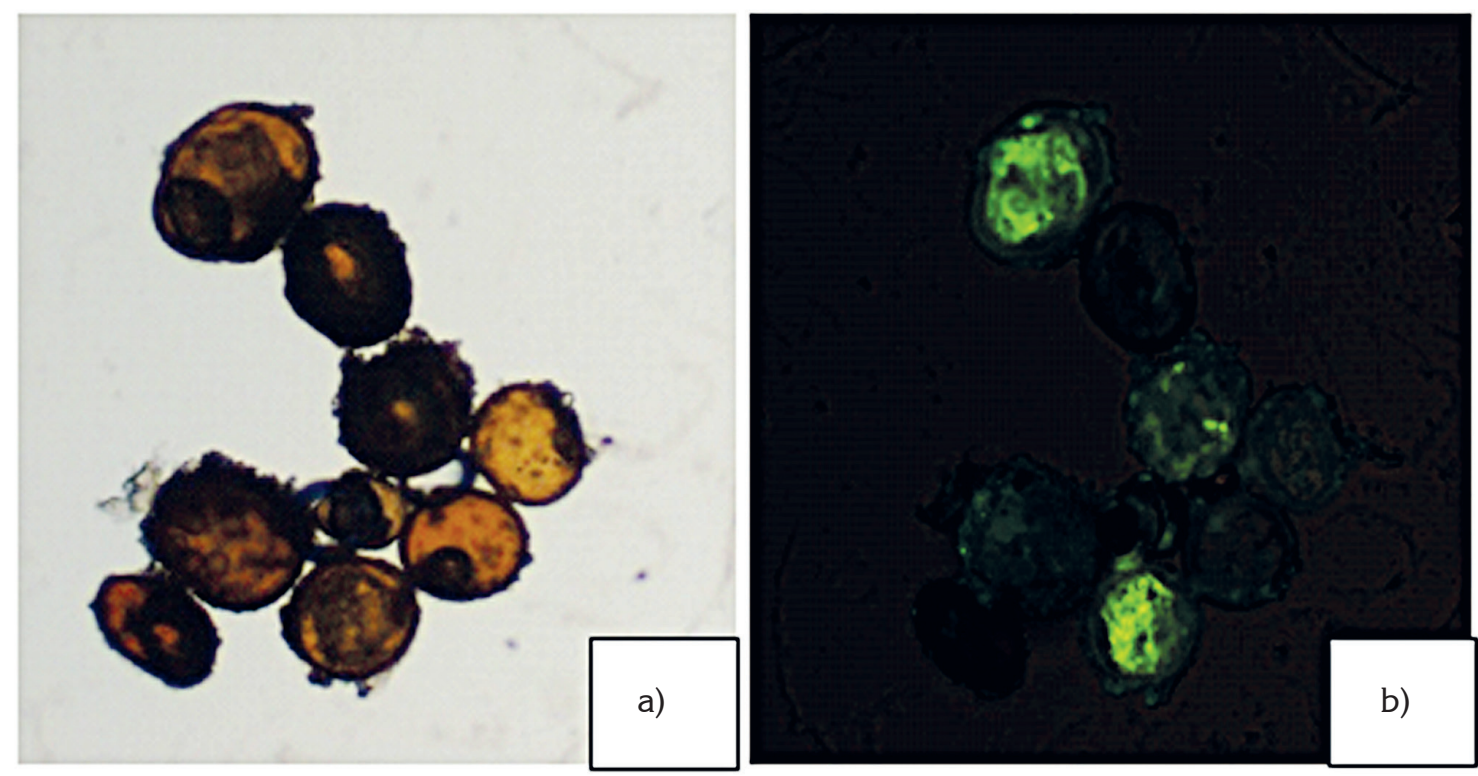

Figura 3. Tinción vital para esporas de Diversispora trimulares, en condiciones de laboratorio. a) Esporas en campo claro (4X); b) Esporas en campo oscuro (4X), esporas viables presentan fluorescencia y muertas no muestran fluorescencia.
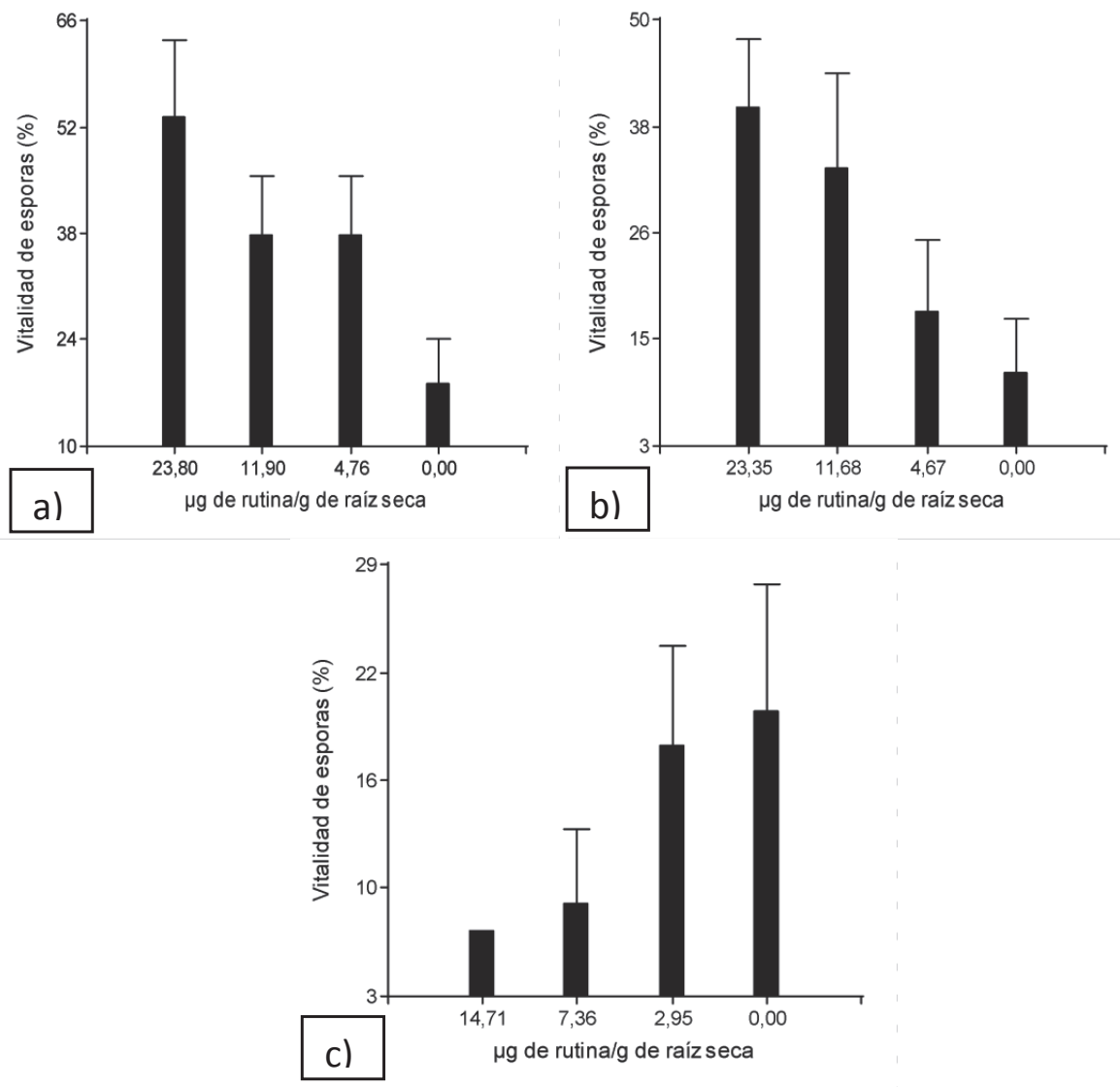

Figura 4. Porcentaje de vitalidad de esporas de Diversispora trimulares, en presencia de exudados radiculares de: a) A. porrum; b) N. glauca; c) B. oleracea, posterior a 30 días de aplicación de los tratamientos, en condiciones de laboratorio. 

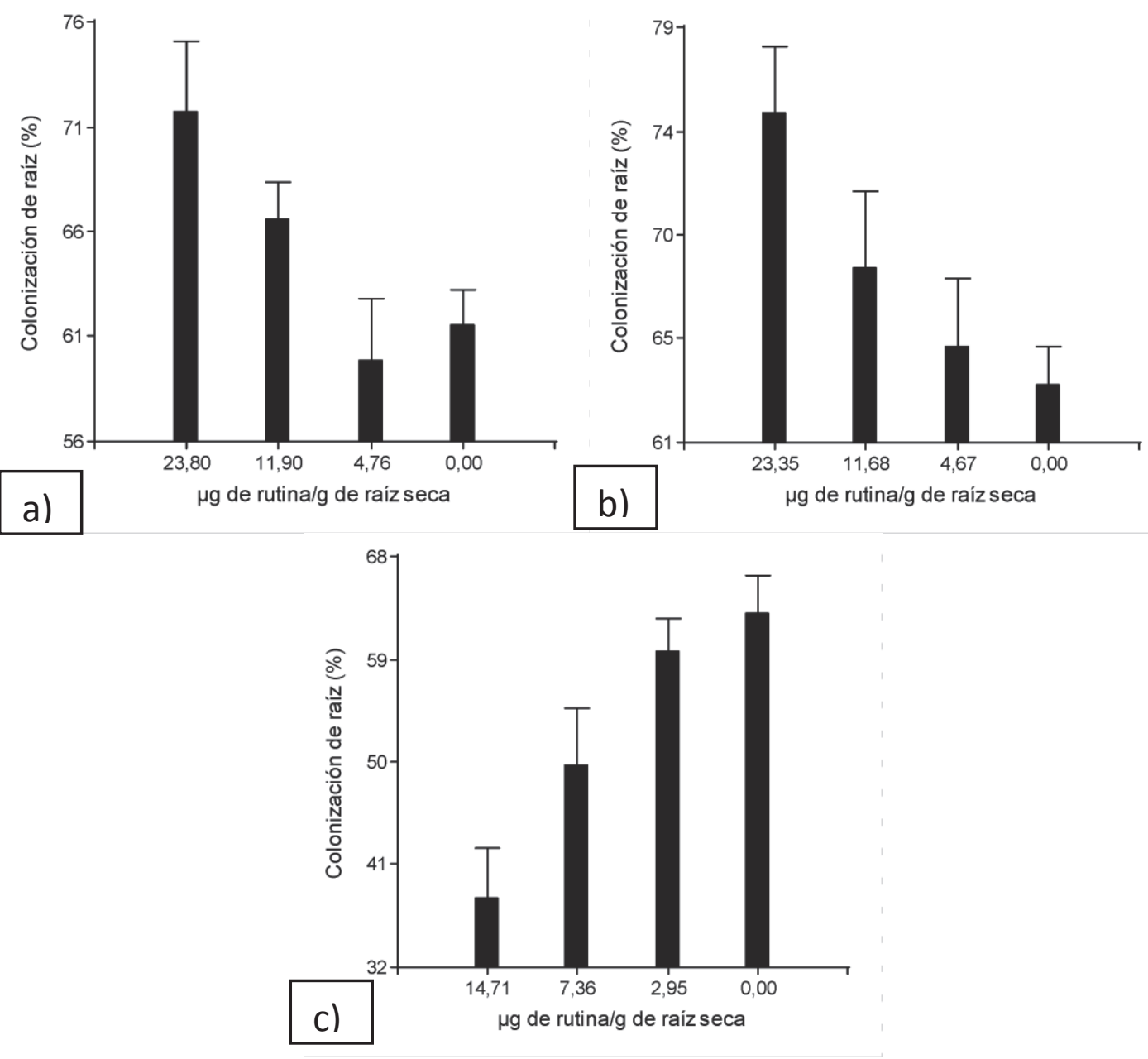

Figura 5. Colonización de raíz de esporas de Diversispora trimulares, en presencia de exudados radiculares, en condiciones de invernadero. a) A. porrum; b) $N$. glauca y c) B. oleracea.

ción y la vitalidad de esporas, a diferencia de crisina, detectada en el exudado radicular de $B$. oleracea, el cual, mostró un efecto inhibitorio en germinación y en colonización radicular de micorriza arbuscular. Adicionalmente, en el experimento en invernadero, se evidencia un efecto sinérgico entre los flavonoides identificados en los exudados radiculares y el $\mathrm{CO}_{2}$, que incrementaron la germinación de esporas y colonización de raíz de micorriza arbuscular, en mayor proporción, que las observadas en condiciones de laboratorio. Todas las respuestas observadas en función de la aplicación de los flavonoides directamente en las esporas y en la interacción planta-HMA son claras respuestas funcionales del efecto que tienen en la estimulación de una rápida germinación y proceso de colonización radicular de micorriza arbuscular en la planta, lo cual, permitirá ventajas en la adquisición de recursos y un efecto directo, en desarrollo de la misma.
Agradecimientos: Agradecemos al Laboratorio de Biotecnología Ambiental de la Universidad Politécnica Salesiana, por su colaboración para realizar los análisis de HPLC; al laboratorio de Microscopía y Biotecnología Humana de la Universidad de las Fuerzas Armadas ESPE, por el apoyo prestado en el transcurso de esta investigación y al Instituto Nacional de Investigaciones Agropecuarias INIAP-Granja Experimental Tumbaco, por facilitar el uso de los invernaderos. Conflicto de intereses: Los autores declaramos que el manuscrito fue preparado y revisado con la participación de todos los autores y que no existe conflicto de intereses que ponga en riesgo la validez de los resultados presentados.

\section{BIBLIOGRAFÍA}

1. ABDEL-LATEIF, K.; BOGUSZ, D.; HOCHER, V. 2012. The role offlavonoids in the establishment of plant roots endosymbioses with arbuscular mycorrhiza 
fungi, rhizobia and Frankia bacteria. Plant Signaling \& Behavior. (Estados Unidos). 7(6):636-641.

2. BANSAL, M.; JAGPAL, R.; MUKERJI, K. 1991. Viability of VAM fungal spores. Recent Advances in Applied Sciences. (Estados Unidos). 6:65-68.

3. BECARD, G.; DOUDS, D.; PFEFFER, P. 1992. Extensive in vitro hyphal growth of vesicular-arbuscular mycorrizhal fungi in the presence of $\mathrm{CO}_{2}$ and flavonols. Applied and Environmental Microbiology. (Estados Unidos). 58(3):821-825.

4. BÜCKING, H.; ABUBAKER, J.; GOVINDARAJULU, M.; TALA, M.; PFEFFER, P.E.; NAGAHASHI, G.; SHACHAR-HILL, Y. 2008. Root exudates stimulate the uptake and metabolism of organic carbon in germinating spores of Glomus intraradices. New Phytologist. (Reino Unido). 180(3):684-695.

5. BUEE, M.; ROSSIGNOL, M.; JAUNEAU, A.; RANJEVA, R.; BECARD, G. 2000. The Pre-Symbiotic Growth of arbuscular mycorrhizal fungi is induced by a branching factor partially purified from plant root exudates. The American Phytopathological Society. (Estados (nnidos). 13(6):693-698.

6. CHABOT, S.; BEL-RHLID, R.; CHENEVERT, R.; PICHÉ, Y. 1992. Hyphal growth promotion in vitro of the VA mycorrhizal fungus, Gigaspora margarita Becker \& Hall, by the activity of structurally specific flavonoid compounds under CO2-enriched conditions. New Phytologist. 122(3):461-467.

7. CESCO, S.; MIMMO, T.; TONON, G.; TOMASI, N.; PINTON, R.; TERZANO, R.; NANNIPIERI, P. 2012. Plant-borne flavonoids released into the rhizosphere: impact on soil bio-activities related to plant nutrition. A review. Biology and Fertility of Soils. (Italia). 48(2):123-149.

8. CESCO, S.; NEUMAN, G.; TOMASI, N.; PINTON, R.; WEISSKOPF, L. 2010. Release of plant-borne flavonoids into the rhizosphere and their role in plant nutrition. Plant and Soil. (Holanda). 329(1-2):1-25.

9. DANESH, Y.R.; NAJAFI, S.; DEMIR, S. 2016. Investigation of the life cycle of arbuscular mycorrhizal fungus (AMF) Glomus intraradices using in vitro culture technique. J. Centenary College Agr. Sci. (Turquia). p.161-167.

10. FERRER, J.L.; AUSTIN, M.B.; STEWART, C.; NOEL, J.P. 2008. Structure and function of enzymes involved in the biosynthesis of phenylpropanoids. Plant Physiology and Biochemistry. (Holanda). 46(3):356-370.

11. FREY-KLETT, P.; GARBAYE, J.A.; TARKKA, M. 2007. The mycorrhiza helper bacteria revisited. New Phytologist. 176(1):22-36.

12. GARCÍA, M.; OVASAPYAN, T.; GREAS, M.; TRESEDER, K. 2008. Mycorrhizal dynamics under elevated $\mathrm{CO}_{2}$ and nitrogen fertilization in a warm temperate forest. Plant and Soil. (Holanda). 303(1-2):301-310.

13. GERDEMANN, J.W.; NICOLSON, T.H. 1963. Spores of mycorrhizal endogone species extracted from soil by wet sieving and decanting. Transactions of the British Mycological society. 46(2):235-244.

14. JAVAID, A.R. 2007. Allelopathic interactions in mycorrhizal associations. Allelopathy Journal. (India). 20(1): 29-42.

15. LUM, M.R.; HIRSCH, A.M. 2003. Roots and their symbiotic microbes: strategies to obtain nitrogen and phosphorus in a nutrient-limiting environment. J. Plant Growth Regulation. (Estados Unidos).21(4):368-382.

16. McGONIGLE, T.P.; MILLER, M.H.; EVANS, D.G.; FAIRCHILD, G.L.; SWAN, J.A. 1990. A new method which gives an objective measure of colonization of roots by vesicular-arbuscular mycorrhizal fungi. New phytologist. 115(3):495-501.

17. MEDINA-MEDRANO, J.R.; ALMARAZ-ABARCA, N.; GONZÁLEZ-ELIZONDO, M.S.; URIBE-SOTO, J.N.; GONZÁLEZ-VALDEZ, L.S.; HERRERA-ARRIETA, Y. 2015. Phenolic constituents and antioxidant properties of five wild species of Physalis (Solanaceae). Botanical Studies. (Taiwan). 56(24):13p.

18. MIEAN, K.H.; MOHAMED, S. 2001. Flavonoid (myricetin, quercetin, kaempferol, luteolin, and apigenin) content of edible tropical plants. J. Agricultural and Food Chemistry. (Estados Unidos). 49(6):3106-3112.

19. MIERZIAK, J.; KOSTYN, K.; KULMA, A. 2014. Flavonoids as important molecules of plant interactions with the environment. Molecules. (Suiza). 19(10):1624016265.

20. MUKHERJEE, A.; ANE, J.M. 2011. Germinating spore exudates from arbuscular mycorrhizal fungi: molecular and developmental responses in plants and their regulation by ethylene. Molecular Plant-Microbe Interactions. (Estados Unidos). 24(2):260-270. 
21. PHILLIPS, J.M.; HAYMAN, D.S. 1970. Improved procedures for clearing roots and staining parasitic and vesicular-arbuscular mycorrhizal fungi for rapid assessment of infection. Transactions British Mycological Soc. 55(1): 158-161.

22. PONCE, M.A.; SCERVINO, J.M.; ERRA-BALSELLS, R.; OCAMPO, J.A.; GODEAS, A.M. 2004. Flavonoids from shoots and roots of Trifolium repens (white clover) grown in presence or absence of the arbuscular mycorrhizal fungus Glomus intraradices. Phytochemistry. (Reino Unido). 65(13):1925-1930.

23. PRIKRYL, Z.; VANCURA, V. 1980. Root exudates of plants. Plant and Soil. (Holanda). 57(1):69-83.

24. REQUENA, N.; SERRANO, E.; OCÓN, A.; BREUNINGER, M. 2007. Plant signals and fungal perception during arbuscular mycorrhiza establishment. Phytochemistry. (Reino Unido). 68(1):33-40.

25. SCERVINO, J.M.; PONCE, M.A.; ERRA-BASSELLS, R.; BOMPADRE, M.J.; VIERHEILIG, H.; OCAMPO, J.A.; GODEAS, A. 2005. Arbuscular mycorrhizal colonization of tomato by Gigaspora and Glomus species in the presence of root flavonoids. J. Plant Physiology. (Reino Unido). 162(6):625-633.

26. SCERVINO, J.M.; PONCE, M.A.; ERRA-BASSELLS, R.; BOMPADRE, M.J.; VIERHEILIG, H.; OCAMPO, J.A.;
GODEAS, A. 2006. Glycosidation of apigenin results in a loss of its activity on different growth parameters of arbuscular mycorrhizal fungi from the genus Glomus and Gigaspora. Soil Biology and Biochemistry. (Reino Unido). 38:2919-2922.

27. SMITH, S.E.; READ, D.J. 2010. Mycorrhizal symbiosis. Ed. Academicpress (Londres). 145p.

28. VIERA, W.; CAMPAÑA, D.; LASTRA, A.; VÁSQUEZ, W.; VITERI, P.; SOTOMAYOR, A. 2017. Micorrizas nativas y su efecto en dos portainjertos de tomate de árbol (Solanum betaceum Cav.). Bioagro (Venezuela). 29(2):105-114.

29. VIERHEILIG, H. 2004. Regulatory mechanisms during the plant - arbuscular mycorrhizal fungus interaction. Canadian J. Botany. (Canada). 82(8):1166-1176.

30. ZHISHEN, J.; MENGCHENG, T.; JIANMING, W. 1999. The determination of flavonoid contents in mulberry and their scavenging effects on superoxide radicals. Food Chemistry. (Reino Unido). 64(4):555-559.

Recibido: Junio 26 de 2017

Aceptado: Agosto 21 de 2017

\section{Cómo citar:}

Ramírez, D.; Naranjo, B.; Duchicela, J. 2017. Estimulación de germinación y colonización radicular de Diversispora trimulares por flavonoides de exudados radiculares de Nicotiana glauca. Rev. U.D.C.A Act. \& Div.Cient. 20(1): $341-351$. 